\title{
Structural characterization of helitrons and their stepwise capturing of gene fragments in the maize genome
}

\author{
Yongbin Dong ${ }^{\dagger}$, Xiaomin Lu ${ }^{\dagger}$, Weibin Song ${ }^{\dagger}$, Lei Shi, Mei Zhang, Hainan Zhao, Yinping Jiao and Jinsheng Lai
}

\begin{abstract}
Background: As a newly identified category of DNA transposon, helitrons have been found in a large number of eukaryotes genomes. Helitrons have contributed significantly to the intra-specific genome diversity in maize. Although many characteristics of helitrons in the maize genome have been well documented, the sequence of an intact autonomous helitrons has not been identified in maize. In addition, the process of gene fragment capturing during the transposition of helitrons has not been characterized.

Results: The whole genome sequences of maize inbred line B73 were analyzed, 1,649 helitron-like transposons including 1,515 helAs and 134 helBs were identified. ZmhelA1, ZmhelB1 and ZmhelB2 all encode an open reading frame (ORF) with intact replication initiator (Rep) motif and a DNA helicase (Hel) domain, which are similar to previously reported autonomous helitrons in other organisms. The putative autonomous ZmhelB1 and ZmhelB2 contain an extra replication factor-a protein1 (RPA1) transposase (RPA-TPase) including three single strand DNAbinding domains (DBD)-A/-B/-C in the ORF. Over ninety percent of maize helitrons identified have captured gene fragments. HelAs and helBs carry 4,645 and 249 gene fragments, which yield 2,507 and 187 different genes respectively. Many helitrons contain mutilple terminal sequences, but only one 3 '-terminal sequence had an intact "CTAG" motif. There were no significant differences in the $5^{\prime}$-termini sequence between the veritas terminal sequence and the pseudo sequence. Helitrons not only can capture fragments, but were also shown to lose internal sequences during the course of transposing.

Conclusions: Three putative autonomous elements were identified, which encoded an intact Rep motif and a DNA helicase domain, suggesting that autonomous helitrons may exist in modern maize. The results indicate that gene fragments captured during the transposition of many helitrons happen in a stepwise way, with multiple gene fragments within one helitron resulting from several sequential transpositions. In addition, we have proposed a potential mechanism regarding how helitrons with multiple termini are generated.
\end{abstract}

\section{Background}

Transposable elements (TEs) not only make up big part of genomes of higher plants, but also play an important role in promoting their genomic diversity $[1,2]$. Helitrons, a new category of DNA TEs, have recently been uncovered by the computational analysis of genomic sequences of $A$. thaliana, O. sativa and C. elegans [3]. Lacking the typical structures that are characteristic of

\footnotetext{
* Correspondence: jlai@cau.edu.cn

† Contributed equally

State Key Laboratory of Agrobiotechnology and National Maize

Improvement Center, Department of Plant Genetics and Breeding, China Agricultural University, Beijing, 100193, China
}

traditional class DNA TEs, helitrons are difficult to be identified. However, helitrons have a "TC" motif on the 5'-terminus and a "CTRR" motif on the 3'-terminus; they also contain a $16-20$ bp palindromic sequence, which can form a hairpin structure of 10-12 bp upstream of the 3'-terminus. In addition, they insert preferentially between adenine and thymidine nucleotides [3,4]. Helitrons are ubiquitous in all studied eukaryotes, such as $A$. thaliana, C. elegans, D. melanogaster, D. rerio, I. tricolor, L. perenne, M. lucifugus, A. gambiae, $M$. Truncatula, N. vectensis, O. sativa, X. maculatus, $S$. bicolor, S. nephelus, and Z. mays [3-12].

\section{Biomed Central}


Helitrons constitute over $2 \%$ of the maize genome. It was estimated that there might be tens of thousands elements in maize inbred line B73 [13,14]. They could capture gene fragments and move around the genome, which leads to gene diversity between the maize inbred lines [15]. Helitrons have contributed the remarkable variation of haplotype in the $B z$ (bronze) genomic locus among different maize inbred lines $[16,17]$. Two helitrons containing hundreds of copies in maize inbred line B73 have been identified [13].

More helitrons and their capture gene fragments have been detected in maize than in A. thaliana and $O$. sativa $[3,13,14,18,19]$. Yang et al. [14] found that over half of the helitrons have contained gene fragments in the B73 genome. They could be from 28 bp to a $7.6 \mathrm{~kb}$ gene fragments in length, and might even include an entire gene sequence [20,21]. According to the results of Du et al. [13] and Yang et al. [14], the helitrons could possess zero to nine gene fragments, which came from 376 and 840 different genes. The gene fragments carried by these elements could also form chimeric genes $[13,20]$. ESTs of helitron sequences have been detected in certain maize tissues [15]. It is possible that some functional genes can be produced from the shuffling of the capture gene fragments.

The mechanism how helitrons capture gene fragments and how they transpose remain unknown. The replication initiator (Rep) protein motif and a DNA helicase (Hel) domain are considered to be the key protein features of rolling circle (RC) processes in bacteria $[3,4,10,22]$. It was postulated that helitrons could mobilize by the RC replication of the "copy-and-paste" model in eukaryotes [4]. Choi et al. [5] found a predicted autonomous element carrying Rep/Hel-TPase and RPATPase in I. tricolor, however, it contained a frameshift and a non-sense mutation. Morgante et al. [15] identified two sequences that contained the conserved RCRep motif and DNA helicase domain in two maize inbred lines. However they both are interrupted by other transposons. Du et al. [13] and Yang et al. [14] proposed that helitrons had amplified within the last 6 million years and could still be active in the modern maize. So far, no intact autonomous element has been discovered in maize $[13,14,19]$.

The full genome sequence of inbred line B73 has been achieved using BAC by BAC sequencing strategy recently [23]. Du et al. [13,24] and Yang et al. [14] have developed methods for identifying helitrons, and mined 2,791 and 1,930 elements, respectively. They had analyzed the extensive distribution, variability and diversity of helitrons in the maize genome. From these studies, certain hallmarks of helitrons in maize have emerged, such as that they preferentially inserted near other ones, but less commonly inserted into certain gene. There were some elements with more than one 5 -termini or 3'-termini. Many helitrons have been shown to carry phosphatase $2 \mathrm{C}$-like gene fragments.

To further understand the characteristics of helitrons as well as the features of their transpositions, we have again developed a set of PERL scripts to search for additional helitrons in the maize genome. A total of 1,649 helitrons have been identified including three putative autonomous elements and two helitrons with high copy number. Our study not only provides a detailed characterization of putative intact automomous helitrons, but also presents evidence to suggest that gene fragment capturing during the transposition of helitrons happened in a stepwise way, with multiple gene fragments within one helitron being the capturing the products of several sequential transpositions. We have also proposed and provided the evidence to support a mechanism regarding how multiple terminal elements are generated.

\section{Results}

\section{Identification of additional helitrons}

To obtain additional helitrons with high confidence, the sequences of 23 published ones [7,15,17,25-27] including twenty helAs and three helBs, were used as query sequences to search against the maize genome sequence by BLASTN. The resulting 248 candidate helitrons were initially identified. To further verify these candidate helitrons, two strategies were used. Firstly, helitron locating in repeated regions could be verified by BLASTN (Additional file 1, Figure S1A) [17]. Secondly, helitrons with multiple copies of high similarity could be verified each other by aligning their sequences together to determine their exact 5' and 3' boundaries (Additional file 1, Figure S1B). Altogether, we obtained 96 validated helitrons by these two methods, including eighty helAs and sixteen helBs. To further confirm these helitrons, we conducted PCR experiments for some selected helitrons. All fourteen that had successful PCR amplification showed variable in sizes of PCR products (Additional file 2, Figure $\mathrm{S} 2$ ), indicating the vacant sites and occupied sites, therefore providing final confirmation for our 96 seed helitrons.

Based on the terminal sequence characteristics of the 96 validated helitrons, a PERL script was designed to identify additional elements in the maize genome. As a result, a total of 1,649 intact elements were obtained. According to a standard previously reported [17], we divided these new elements into two different families, which including 1,515 helAs and 134 helBs (Additional file 3, 4, Table S1, S2). The size of these elements ranged from $128 \mathrm{bp}$ to $20,874 \mathrm{bp}$; the average length was 6,357 bp for helA, and 4,629 bp for helB. Overall, $82.7 \%$ $(1,253 / 1,515)$ of helA sequences were less than $10 \mathrm{~kb}$ in length. Similarly $94.8 \%(127 / 134)$ helB were less than 10 
kb. HelAs with the length of over $10 \mathrm{~kb}(22.5 \%$; 59/262) and all 7 helBs with the length of over $10 \mathrm{~kb}$ were classified as putative "autonomous" helitrons if they do not contain other long transposons such as retrotransposon.

HelAs had a conserved sequence of the $24 \mathrm{bp}$ at the 5 'terminus and $28 \mathrm{bp}$ at the 3 '-terminus including palindromic structures. HelBs had conservative sequences for $28 \mathrm{bp}$ and $32 \mathrm{bp}$ at the 5 '-terminus and 3'-terminus, respectively (Additional file 5, Figure S3). The 5'-terminus of helBs was significantly different from those of helAs.

\section{Putative autonomous helitrons}

In general, the helitrons that encode replication initiator (Rep) motif, DNA helicase domain and a possible replication A protein 1 (RPA1)-like motif in plants, are considered as putative autonomous ones [4]. To find potential autonomous helitrons, all helAs sequences of over $10 \mathrm{~kb}$ and helBs of over $5 \mathrm{~kb}$ were carefully annotated. Two sequences, named ZmhelA1 (AC208648.2, 14,632 bp) and ZmhelB2 (AC212020.2, 12,217 bp) respectively were qualified as putative autonomous elements. ZmhelA1 and ZmhelB2 all contained conserved Rep motif and DNA helicase domain without frameshift (Figure 1A, B, C, Additional file 6, Table S3). Those conserved domains were reported to be essential for DNA replication and for unwinding double stranded DNA in other prokaryotic and eukaryotic species $[3,5,10]$. The putative autonomous ZmhelA1 also contained a putative RPA remnant before the Rep motif (Figure 1A), although the RPA sequence had a very low sequence homologous with that of $A$. thaliana and $O$. sativa [3]. In addition, ZmhelA1 also carried eight predicted gene fragments. ZmhelB2 possessed three putative single strand DNA-binding domains (DBD)-A/-B/-C of RPA1 following the helicase domain in the ORF (Figure $1 \mathrm{~A})$, which were in the same orientation as the Rep/ Helicase gene. ZmhelB2 also carried two postulated gene fragments. Based on these structural characteristics, autonomous helitrons in maize could be at least divided into two types, a result that was consistent with the neighbor-joining phylogeny analysis (Figure 2).

To obtain additional putative "autonomous" elements, the RPA-like and DNA helicase of $A$. thaliana and $O$. sativa $[3,5]$ were used to search against maize genome by TBLASTN respectively. Then the obtained sequences were extended $10 \mathrm{~kb}$ each in the 5'-terminus and 3'-terminus respectively. Finally, the obtained putative autonomous helitrons were annotated by Fgenesh (http:// linux1.softberry.com/berry.phtml). As a result, five putative autonomous helBs were identified by this homolog searching approach. One of the five putative autonomous helBs, ZmhelB1 (AC200867.3) with the length of $12,992 \mathrm{bp}$, also encoded an intact ORF as ZmhelB2 with potentially functional Rep motif, a DNA helicase domain and a RPA1 motif without frameshift (Figure 1A, B, C, Additional file 6, Table S3). These two putative autonomous helBs have similar structural characteristics as that reported by Morgante et al. [15].

\section{Helitrons of multiple terminal sequences and of high copy number}

Our result showed that $28.7 \%$ of helAs had contained multiple terminal structures. We called the internal terminal sequences as the pseudo terminus (Figure 3A, $B, C)$. Through multiple sequence alignment, we found that the real 3'-terminus of helitrons contained highly conserved "CTAG" motif, but not at the pseudo 3'-terminus of elements with multiple 3'-termini (Figure 3D). One hundred helAs with multiple 3'-termini were randomly sampled to analyze structure of their pseudo 3'termini, the result showed that 99\% (99/100) of the internal 3' end sequence had a pseudo 3'-terminus with no intact "CTAG" motif. However, we did not find any multiple terminal sequences in the 134 helBs.

Based on the sequence characteristics of pseudo 3'-termini that we obtained, the following consensus sequence model was defined: "CCGT[ATCG]GCA[AT]CGCACG [AG] $\{2\}[$ ATCG] $\{6,8\} C$ TAT". By searching against the maize genome sequence according to the model, 662 pseudo 3'-termini sequences were obtained. Ten sequences were randomly selected from these newly identified pseudo 3'-termini, and the intact 3'-termini structures were shown within $10 \mathrm{~kb}$ downstream. It was ubiquitous that the pseudo 3'-termini we identified had no intact "CTAG" motif in maize. Using the same methods, we found that $17.6 \%$ of helAs also had multiple 5 -termini. However, there were no distinct differences between the pseudo 5'-terminal sequence and the true 5'-terminal one.

Helitrons with many copies have been previously identified in inbred line B73 $[13,14]$. Here we found two additional elements with high copy number. Two of the helAs, named helitron_mc1 (AC186621.4, $1615 \mathrm{bp}$ ) and helitron_mc2 (AC188746.2, $2683 \mathrm{bp}$ ), possessed 50 and 54 copies with a high stringent criteria (coverage $>95 \%$ and identities $>95 \%$ ), respectively. Using a more relaxed set of criteria (sequence identity $>80 \%$, size $>200 \mathrm{bp}$ ), there were 2,450 and 5,103 copies, respectively (Table 1 , Additional file 7, 8, Table S4, S5). Helitron_mc1 had over $85 \%$ identities in 1,300 bp of the 3'-end sequence with helitron_mc2. It is possible that helitron_mc2 have evolved from helitron_mc1. In addition, helitron_mc2 also possessed two pseudo 3'-termini structures (Figure 4).

\section{Gene fragments captured by helitrons}

In order to analyze the gene fragments carried by helitrons, all detected elements were searched against the nonredundant protein (nr) database using the BLAST 
A. Putative autonomous helitrons:

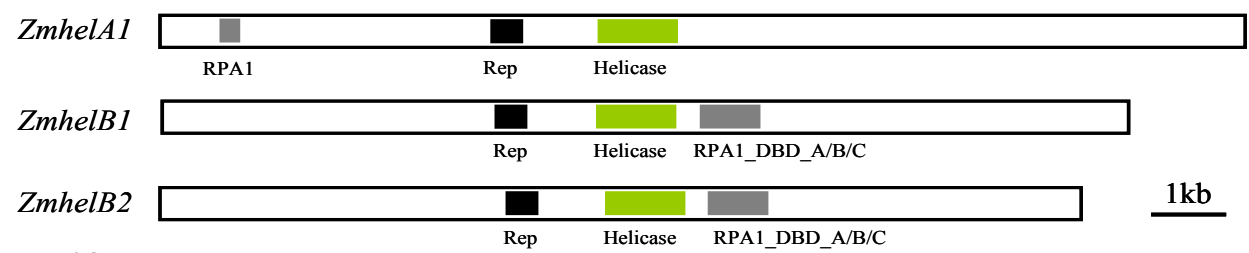

\section{B. Rep motif:}
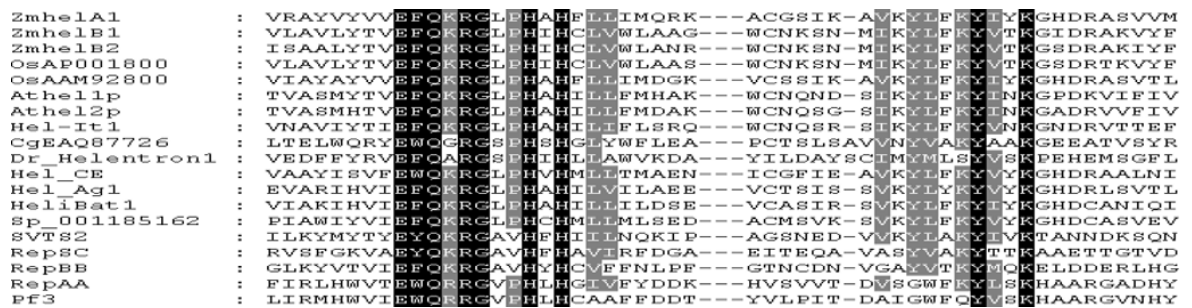

\section{DNA helicase domain:}
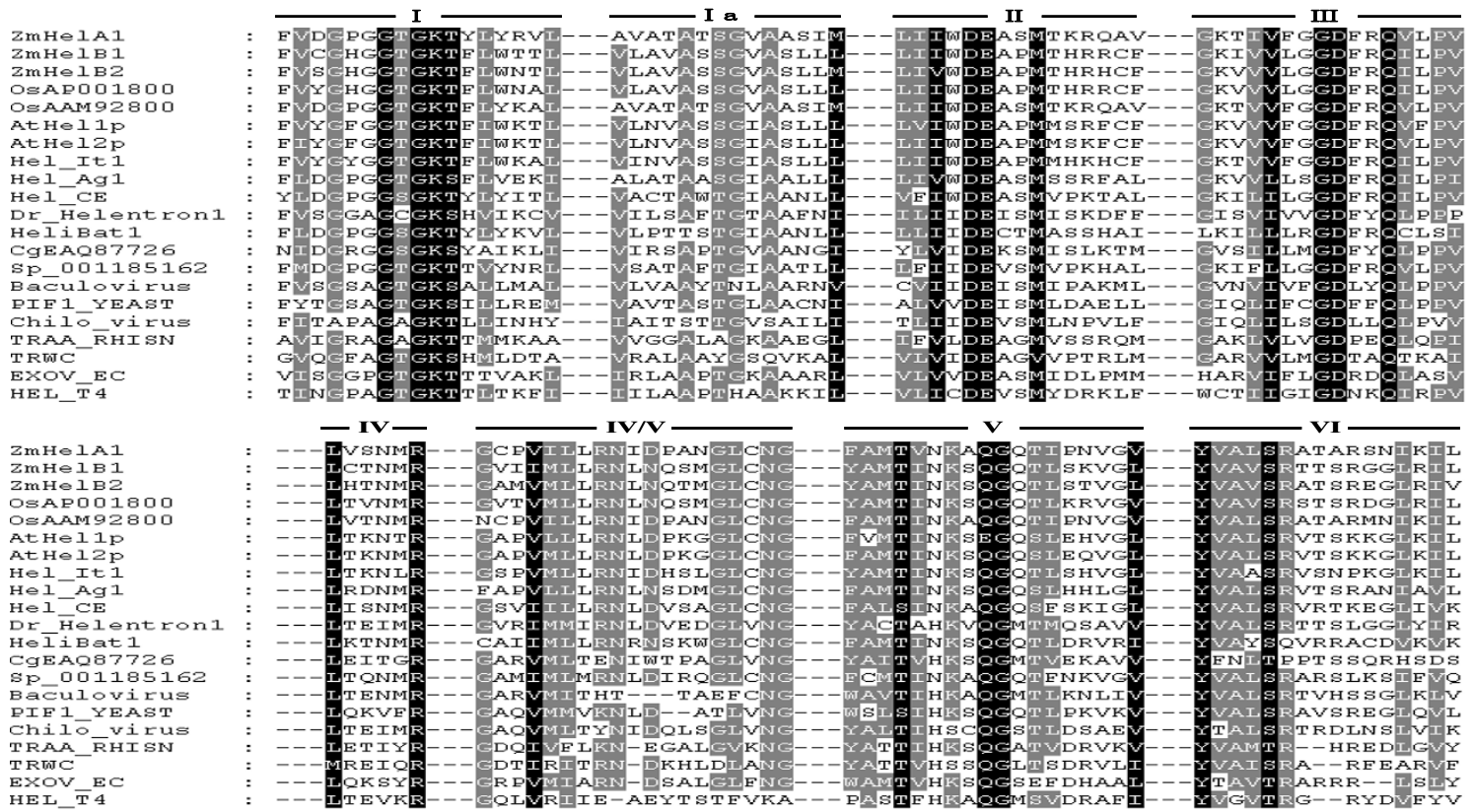

Figure 1 Gene structure, the Rep protein motif and DNA helicase domain of putative autonomous ZmhelA and ZmhelB. A. A schematic diagram of putative autonomous ZmhelA and ZmhelB showing the Rep, helicases and RPA domain. B and C. Multiple sequence alignments of the Rep motif and DNA helicase domain. Sequences from other species were aligned with ZmhelA1, ZmhelB1 and ZmhelB2. Ce, C. elegans; Ag, Anopheles gambiae; Os, Oryza sativa; At, A. thaliana; It, Ipomoea tricolor; Dr, Danio rerio; Cg, Chaetomium globosum; Sp, Strongylocentrotus purpuratus; SVTS, Spiroplasma plectrovirus (AAF18311); Rep_SC, Streptomyces cyaneus plasmid (BAA34784); Rep_BB, Bacillus borstelensis plasmid (BAA07788); Rep_AA, Actinobacillus actinomycetemcomitans plasmid (AAC37125); Pf3, Pseudomonas aeruginosa bacteriophage (AAA88392); Baculovirus (NP047686); Yeast (P07271); CHilo (AAD48149); TRAA_RHISN (P55418); TRWC (S43878); EXOV_EC (P04993); HEL_T4 (P32270) [10].

program. Most of helitrons with a size of less than $1 \mathrm{~kb}$ (64.7\%) did not contain any gene fragment. Most of elements with lengths from $1 \mathrm{~kb}$ to $2 \mathrm{~kb}(90 \%)$ had only obtained one gene segment. The number of capture gene fragments by the helAs ranged from 0 to 12, with a mean value of 3 . Most helAs (82.1\%) carried between
1 and 5 gene fragments. All of the helBs held no more than five gene fragments, with an average of 1.8. The majority of helBs (82\%) acquired 1 to 3 gene fragments (Figure 5).

A total of 4,645 gene fragments were carried by the helAs, which encoded 2,507 proteins (Additional file 9, 


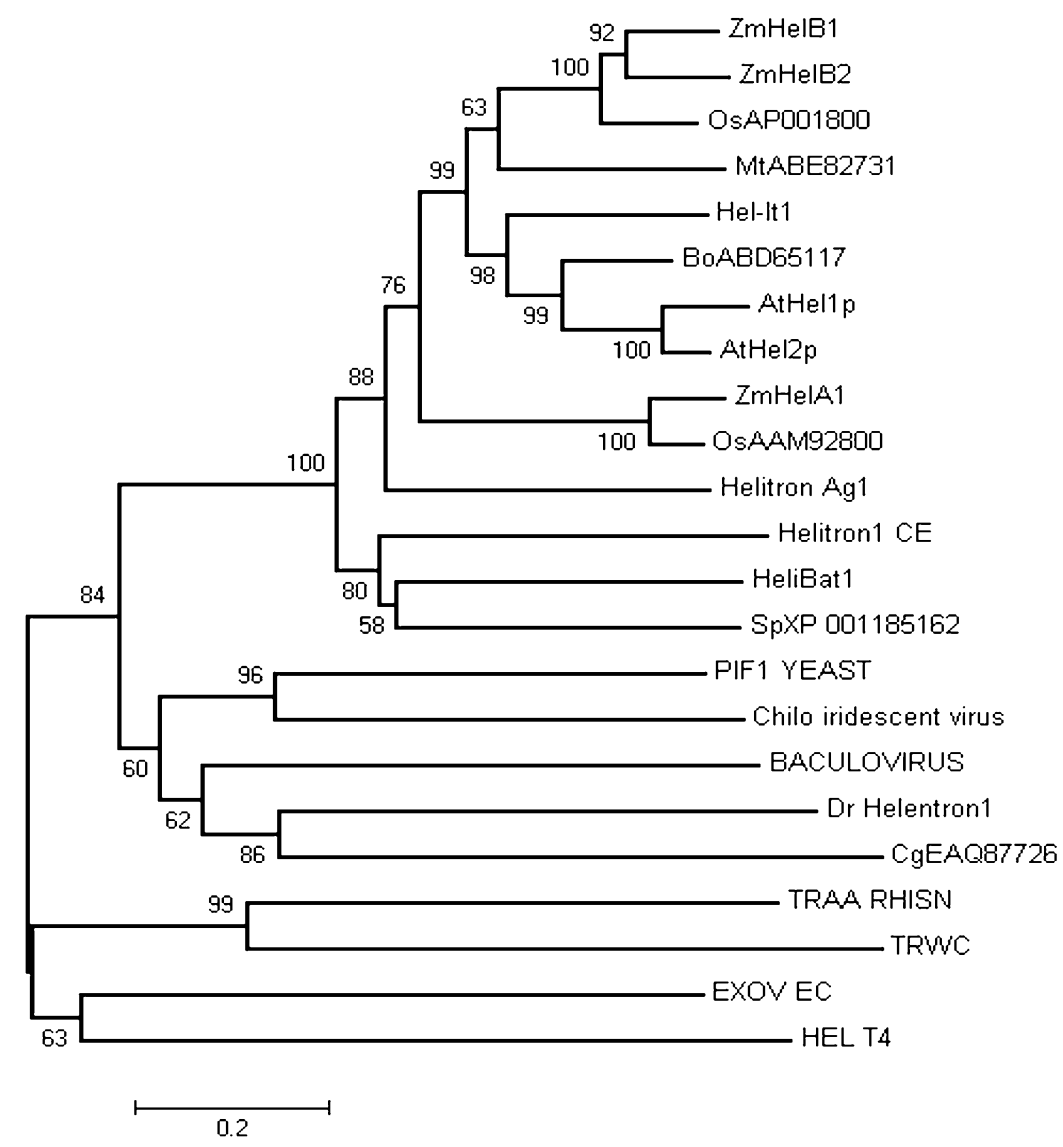

Figure 2 A phylogenetic tree of DNA helicase of putative autonomous helitrons of maize and other species. The phylogenetic tree was constructed by the neighbor-joining method using MEGA4 software [32] with 1,000 bootstrap replicates, the bootstrap scores < 50\% were deleted. The accession numbers and names of the putative helicases of other species were abbreviated as shown in Figure 1, with the addition of the following: Bo, Brassica oleracea (ABD65117); Mt, Medicago trunculata (ABE82731) [10].

A. Ac177883 a
B.

Figure 3 Helitrons of multiple termini and their sequence characteristics of $\mathbf{3}^{\prime}$-termini in maize. A, B and C. Helitrons with multiple termini. The black and red boxes indicate $5^{\prime}$-termini and $3^{\prime}$-termini of helA respectively. D. Alignment of the pseudo $3^{\prime}$-termini and the real ones in Figure $3 A, B, C$. 
Table 1 Copy numbers of helitron_mc1 and helitron_mc2.

\begin{tabular}{|c|c|c|c|}
\hline Name & $\begin{array}{l}\text { Size } \\
\text { (bp) }\end{array}$ & Standard & $\begin{array}{c}\text { Copy } \\
\text { number }\end{array}$ \\
\hline \multirow[t]{4}{*}{ helitron_mc1 } & 1,615 & $\begin{array}{l}\text { coverage }>95 \% \text { and } \\
\text { identities }>95 \%\end{array}$ & 50 \\
\hline & & $\begin{array}{c}\text { coverage }>90 \% \text { and } \\
\text { identities }>90 \%\end{array}$ & 88 \\
\hline & & $\begin{array}{l}\text { coverage }>85 \% \text { and } \\
\text { identities }>85 \%\end{array}$ & 332 \\
\hline & & $\begin{array}{c}\text { coverage }>200 \text { bp and } \\
\text { identities }>80 \%\end{array}$ & 2450 \\
\hline \multirow[t]{4}{*}{ helitron_mc2 } & 2,683 & $\begin{array}{l}\text { coverage }>95 \% \text { and } \\
\text { identities }>95 \%\end{array}$ & 54 \\
\hline & & $\begin{array}{l}\text { coverage }>90 \% \text { and } \\
\text { identities }>90 \%\end{array}$ & 89 \\
\hline & & $\begin{array}{l}\text { coverage }>85 \% \text { and } \\
\text { identities }>85 \%\end{array}$ & 94 \\
\hline & & $\begin{array}{c}\text { coverage }>200 \mathrm{bp} \text { and } \\
\text { identities }>80 \%\end{array}$ & 5103 \\
\hline
\end{tabular}

Table S6). There were 229 helAs that had captured a near identical fragment of phosphatase (type) 2C-like protein (ACG41393.1) [13,14], the same gene fragment found in helitron_mc1 and helitron_mc2. Different members of the phosphatase (type) $2 \mathrm{C}$ family protein were also captured by other helAs, such as ACF84978.1 (48 hits), AAQ06294.1 (29 hits) and ACF83293.1 (19 hits). It is possible that the phosphatase (type) $2 \mathrm{C}$-like protein carried by the helAs could have been amplified previously [13].

A total of 249 gene fragments coming from 187 proteins have been captured by helBs (Additional file 10, Table S7). There were 6 helBs that contained a same gene fragment (ACG47094.1). Our results suggest that helitrons do not have a bias in capturing gene fragments.

\section{Step by step capturing of gene fragment}

Many helitrons have captured several gene fragments. Some of the gene fragments are apparently even from

A.

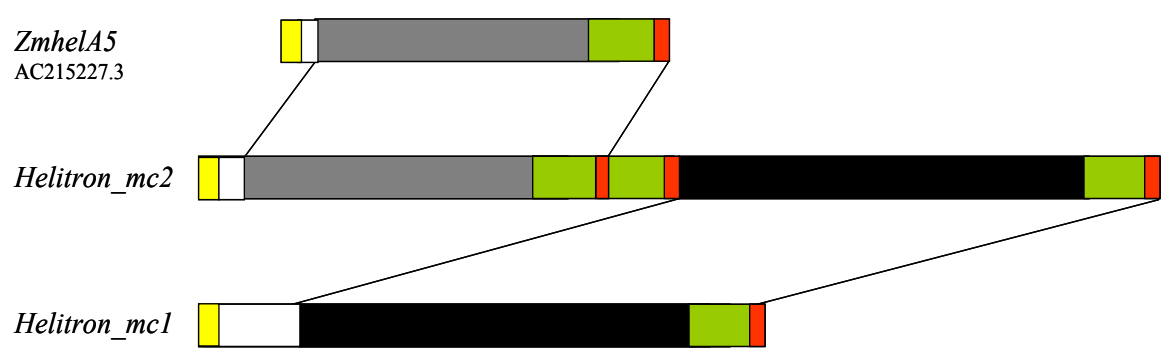

B. ZmhelA5

Helitron_hypo 2

Helitron_mc1

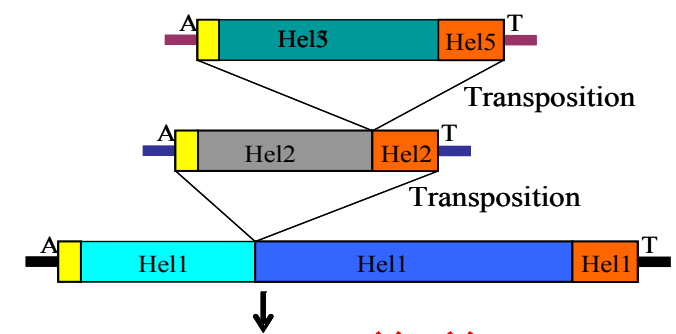

C.

TC

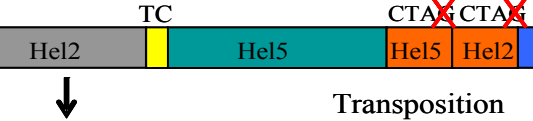

D. Helitron_mc2

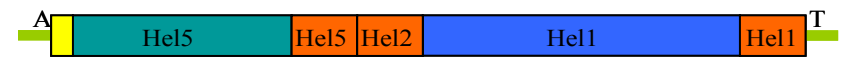

\section{$\left.\begin{array}{l|l|l}\mathrm{A} & \text { Hel1 } & \text { Hel2 }\end{array}\right]^{\mathrm{T}}$ Seq_remain}

Figure 4 The putative evolutionary relationship among helitron_mc1, helitron_mc2 and ZmhelA5. A. Structural information of three helitrons identified in the maize genome. The size of helitron_mc1, helitron_mc2 and ZmhelA5 were 1,617 bp, 2,683 bp and 1,111 bp, respectively. Sequences between two lines had identities $\geq 85 \%$. B, C and D. The hypothesized evolutionary path for helitron with multiple 3' termini. B. zmhelA5 inserted into helitron_hypo2 to form new helitron. Then new helitron_hypo2 inserted into helitron_mc1 (hel1) to form the C state. C to D: The nested intermediate helitron transposed starting from the 5'-terminus of ZmhelA5 to generate helitron_mc2 with three 3'-termini, leaving a remnant with two $5^{\prime}$-termini. 


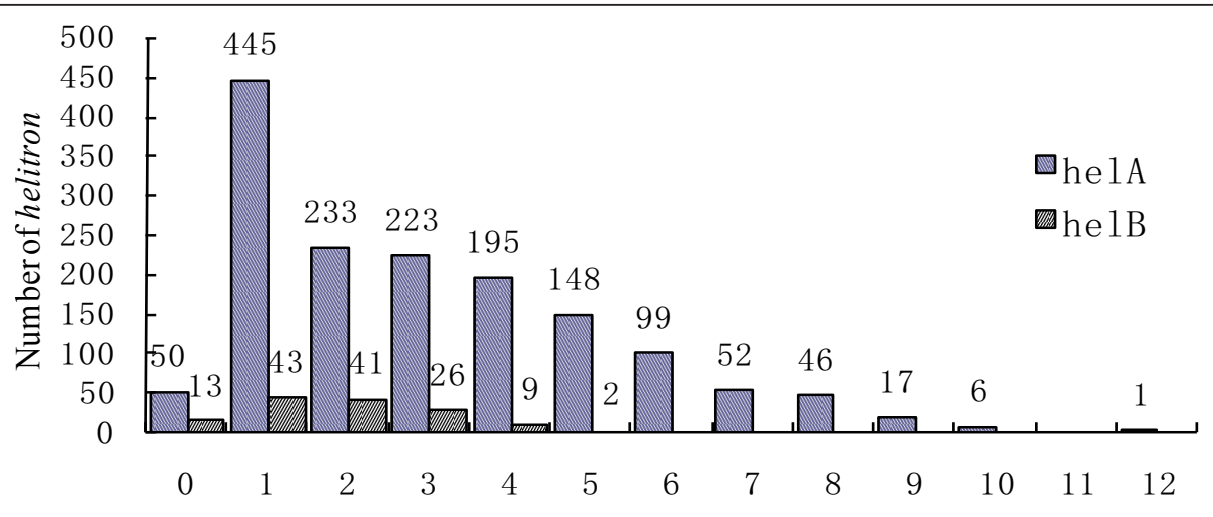

Distribution of the number of gene fra gments captured per helitron

Figure 5 Distribution of the number of gene fragments carried by helAs and helBs. X-axis, the number of gene fragments; $y$-axis, the number of helAs and helBs.

different chromosomes of the maize genome. How can a single helitron capture a number of gene fragments originally located in several different loci of the genome is a big puzzle thus far. Extensive sequence alignment analyses showed that there was high level but fragmented sequence homology within their captured gene fragments among a number of newly identified helitrons. For example, several captured gene fragments of ZmhelA3 (362 bp, AC197568.2) were shown to have high sequence similarity with multiple captured fragments of ZmhelA2 (1,728 bp, AC216828.1), ZmhelA4 (1,520 bp, AC213839.3), helitron_mc1 and helitron_mc2
(Figure 4A, 6A) respectively. All these four elements have near identical first $25 \mathrm{bp}$ of their 5 -termini and last $30 \mathrm{bp}$ of their 3'-termini. Interestingly, ZmhelA3 and ZmhelA2 have over 95\% identity from 5' to 3' end, excepting one insertion in the middle for ZmhelA2. Therefore, ZmhelA2 can be explained by having captured a 1,366 bp gene fragment and having inserted into 25 bp of its 5'-termini of its ancestral element (ZmhelA3). In the same way, ZmhelA4 and helitron_mc1 showed high sequence similarity (more than $85 \%$ ) with the 193 bp of the 3'-terminus of ZmhelA3. Detailed analysis indicated that, starting from an ancestral element

A.

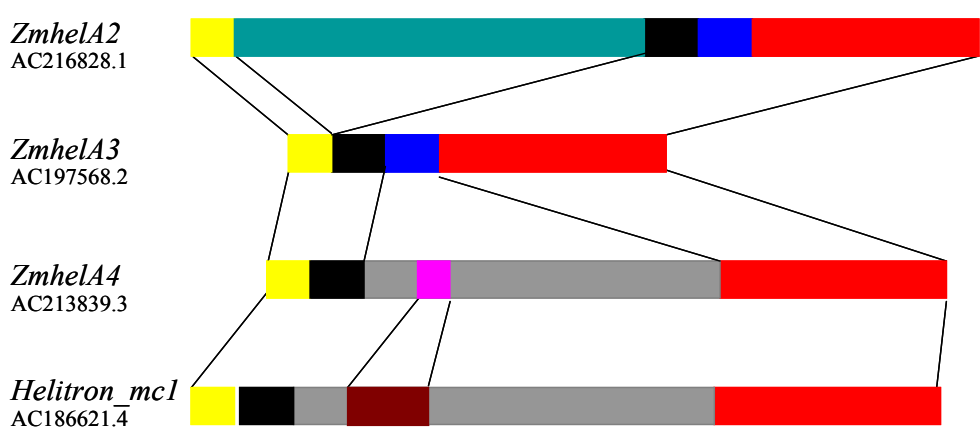

B.

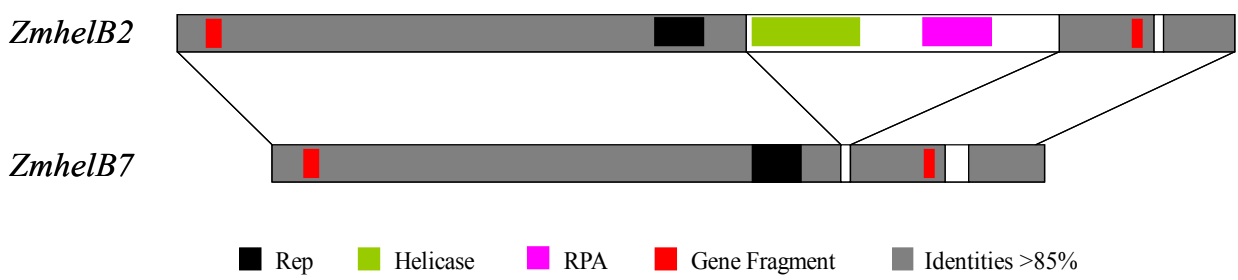

Figure 6 Sequence homology of related helitrons. A. Fragmented sequence homology of four related helitrons. Accession number where the helitrons were identified were shown on the left, segments with the same colors have sequence identities $>85 \%$. B. Structural relationship between $Z$ mhelB7 and the putative autonomous ZmhelB2. 
that is missing only one internal gene fragment (shown in blue as Figure 6A) from ZmhelA3, the ZmhelA4 and helitron_mc1 can both be generated by capturing different gene fragments over several steps of transposition. Our result strongly suggested that the gene fragments captured by helitrons happened in sequential fashion, with each step of transposition likely capturing one gene fragments. In fact, such a stepwise gene capturing capacity will provide endless opportunity to shuffle gene fragments originating from all over the genome.

ZmhelB7 (AC186647.3, AC212020.4) might have evolved from ZmhelB2. ZmhelB7 and ZmhelB2 have the same terminal sequences, but the former lacked the DNA helicase domain and the replication protein A (RPA)-like fragments that were found in ZmhelB2 (Figure 6B). This indicated that helitrons could lose the internal sequence during the process of transposition in maize.

\section{Discussion}

Helitrons are particularly complex in the maize genome $[13,14,28]$. A total of 1,649 elements were obtained based on the terminal sequence characteristics of elements in this research. Du et al. [13] and Yang et al. [14] identified 2,791 and 1,930 intact elements in the maize genome, which overlapped $52.46 \%$ and $34.45 \%$ with our result respectively (Additional file 11, 12, Table S8, S9). The differences among these three searching programs are mainly due to the parameters used in the respective perl scripts. For example, the script used by Du et al. [13] only aimed to identify helAs, while script used in this study is intended to cover both helAs and helBs. Additionally, Du's script and that of the current study have also differed in a number of searching criteria which leaded to a number of specific helitrons being identified by each script. Based on previous estimation [15], there are still a large number of helitrons in maize B73 genome have not been identified. Due to the unique structure of helitrons, it is still very difficult to unambiguously identify all these elements. With more seed helitrons available, a more accurate script could be generated which would drastically increase the number of elements being identified in the B73 genome.

\section{Putative autonomous helitrons}

All helitrons that have been identified so far in the maize genome are nonautonomous [13,14]. In fact, truly autonomous elements have not been found in eukaryotic species to date. In a spontaneous pearly-s mutant of I. tricolor, Choi et al. [5] found that a putative autonomous helitron containing Rep/Hel-TPase and RPATPase, but it had a frameshift and a nonsense mutation. Morgante et al. [15] identified two sequences contained Rep motif and DNA helicase domain. However they both are interrupted by other transposons. Three putative autonomous helitrons found in this research have contained intact Rep motif and DNA helicase domain, the same as those found in A. thaliana and O. sativa [3]. We also detected other four helBs with the conserved Rep motif and the DNA helicase domain, however, their ORF were either having frameshift or incomplete (Additional file 6, Table S3). Although we can not confirm that these three putative autonomous helitrons are actually function as autonomous element at present, the presence of these three putative autonomous sequences with intact ORF in the B73 genome is strongly suggested that true autonomous helitrons could exist in modern maize.

$Z$ mhelA1 had a putative RPA remnant before the Rep motif. ZmhelB1 and ZmhelB2 possessed an intact RPA1-like domain following the helicase domain in the same ORF respectively. Choi et al. [5] speculated that Rep/Helicase were ubiquitous in eukaryotes, and could play a more important role in the helitrons transposition than RPA1. The structural characteristics of putative autonomous elements in A. thaliana, C. elegans, I. tricolor, M. lucifugus, O. sativa and $Z$. mays were carefully analyzed [3,5,10,15] (Additional file 13, Table S10). The putative autonomous elements in animal only contain the conserved Rep motif and DNA helicase domain. The putative autonomous elements in plants all contain RPA-DBD-A/-B/-C before Rep motif or after helicase domain, except for the conserved Rep motif and DNA helicase domain. If ZmhelA1 indeed function as an autonomous element, then it would suggest that RPA1 is not an indispensable feature for helitrons transposition. The putative autonomous helitrons in plants can be divided into two types. One is RPA-DBD-A/-B/-C, following the successive Rep motif and DNA helicase domain in two different ORF respectively. The second contains the Rep motif, DNA helicase domain and RPA$\mathrm{DBD}-\mathrm{A} / \mathrm{-B} / \mathrm{-C}$ in their appropriate order in the same ORF.

\section{Generation of helitron with multiple termini from nested helitrons}

Most helitrons in the maize genome were found to be small sizes. About $80 \%(1,253 / 1,515)$ helAs were between $100 \mathrm{bp}$ and $10 \mathrm{~kb}$ in length, and 94.8\% (127/ 134) helBs ranged from $600 \mathrm{bp}$ to $10 \mathrm{~kb}$ in this research. Yang et al. [14] identified 1,930 elements, of which $95.4 \%(1,841 / 1,930)$ were less than $10 \mathrm{~kb}$ in length. The finding of helitrons with multiple copies suggests that they do not always capture gene fragments in the process of transposition.

There were $28.7 \%$ helAs that possessed multiple terminal structures as shown by Du et al. [13]. The pseudo 3'-termini sequences had damaged "CTAG" motif 
comparing with the real 3'-termini. We found that the pseudo 3'-termini structures were ubiquitous in maize inbred line B73. HelAs had preference to insert near to or inside other helitrons [14], which could have caused to form multiple terminal sequences inside them. Genomic evolution or transpositions could have caused an intact terminal structure to turn into a pseudo 3'-terminus (Figure 4B, C, D). Yang et al. [14] reported that helitrons could recognize a new 3'- or 5'-terminus site to form a new element in A. thaliana. Du et al. [13] found that the 3 '-termini sequences were more variable than the 5'-termini ones.

The evolutionary pathway of helitrons with shared capture gene fragments can be deduced according their different combination of their capture gene fragments (Figure 6A, B). We detected two elements with multiple copies, helitron_mc1 and helitron_mc2, the latter possessed two pseudo 3'-termini structures (Figure 4). There was a high similarity in the 5'-terminal sequence of helitron_mc2 and ZmhelA5 (AC215227.3). Helitron_mc1, helitron_mc2 and ZmhelA5 had one, three and one fragment respectively, which are highly homologous to $193 \mathrm{bp}$ of the 3'-terminus of ZmhelA3 (Figure 4.A). According to these observations, helitron_mc2 might have evolved from helitron_mc1 and ZmhelA5 $[29,30]$. The detail of the hypothesized evolution path for helitron_mc2 is shown in Figure 4B, C, D. ZmhelA5 were inserted into helitron_hypo2 (a hypothesized intermediate). Then helitron_hypo2 carrying ZmhelA5 inserted into helitron_mc1 to form nested heltrons. Eventually helitron_mc2 was generated by further transposition starting from the 5 '-end of ZmhelA5 while including the rest of three 3' ends. The intact 3' end "CTAG" motif can be mutated either before or after the generation of helitron_mc2. As there exist a large number of nested retrotransposons [31], there can be a lot of nested helitrons in the maize genome. The later is then served as intermediate to give rise to many helitrons of multiple termini seen in the B73 genome.

\section{Conclusions}

Helitrons in the maize genome are variable size. When the elements transposed, they could sometimes capture gene fragments or lose their internal sequence. Gene capturing of helitrons can happen in a stepwise mode through sequential transpositions. Three putative autonomous helitrons were discovered in maize with intact replication initiator (Rep) motif and a DNA helicase (Hel) domain, similar to those identified in other species. Therefore, it is possible that active autonomous elements exist in modern maize. Our study also indicated that helitrons with multiple termini can be generated from nested helitrons.

\section{Methods}

\section{Identification of new helitrons}

We initially used 23 published helitrons including 20 helAs and 3 helBs [7,15,17,25-27] (downloaded from http://genomecluster.secs.oakland.edu/helitrons/). They were used as query sequences to search against the maize genome sequence by BLASTN. Searches were conducted according to the following criteria for the termini of candidate helitrons: 5' match coverage $>25 \mathrm{bp}$, identities $>70 \%$; 3' match coverage $>25$ bp, identities $>80 \%$.

Two candidate elements with less than $20 \mathrm{~kb}$ between them were regarded as a single helitron. We initially obtained 248 candidate helitrons. A single element that had inserted into highly duplicated regions could be verified by BLASTN (Additional file 1, Figure S1A) [17]. Secondly, helitrons with multiple copies of high similarity are verified by aligning their sequences together to determine their exact 5' and 3' boundaries (Additional file 1, Figure S1B). Through these two methods, we finally validated 96 helitrons. Then primers of fourteen sequences of validated 96 elements were designed to the flanking regions upstream and downstream of the inserted element to verify the putative helitrons, to see the vacant sites and occupied sites displayed by different PCR bands in a set of 12 inbred lines (Additional file 2, Figure S2).

A PERL script was then written based on terminal characteristics of 96 validated elements to search against the sequence database of the inbred line B73. We applied two steps to identify helitrons more reliably, firstly using the following search criteria: helA 3'-end, CCCGT. $\{6,8\}$ ACG[GA][GA].\{6,8\}CTAGT; helA 5'-end, ATC[TC][ATCG]TA[TC]TA[TCA][ATCG]\{5,6\}AAG; helB 3'-end, CGCC. $\{5,7\}$ GGCG. $\{8,10\}$ CTAGT; helB 5'end, ATC $[$ ATCG] $\{7,8\}$ TTAAAA.

According to the search results and the validated criteria mentioned above, we searched the genomic sequences again using the stricter criteria as follows: helA 3'-end, CCGT.GCA[AT]CGCACG[GA] $\{2\} .\{7\}$ CTAGT helA 5'-end, ATCT[ATCG]TACTAC.\{5\}A helB 3'-end, GCGCCC.\{4\}GGGCGC. $\{8\} C T A G T$ helB 5'-end, ATC[TGA].\{4\}[TC][AC]TTAAAA A total of 1,649 intact elements were identified by this way. Helitrons with multiple termini were searched against the maize genome according to the following criteria, but avoiding the 3 -termini of elements that ended in a guanine base: CCGT[ATCG]GCA[AT]CGCACG[AG] $\{2\}[$ ATCG] $\{6,8\}$ CTAT.

\section{Sequence analysis and annotation}

Local BLAST software (blast-2.2.16) was used to align the sequences. A neighbor-joining phylogeny $(1,000$ 
bootstrap replications) was built for the helicases of different species by the Molecular Evolutionary Genetics Analysis (MEGA) 4.0 software [32]. CLUSTALX 2.0 software was used to align sequence. Identified helitrons were annotated by FGENESH (http://linux1.softberry. com/berry.phtml).

The sequences of newly identified helitrons $(1,649)$ were used to blast against the nr protein sequence database in NCBI (http://www.ncbi.nlm.nih.gov/). Information about the quantity, location and annotation of capture gene fragments was obtained from the blast results.

\section{PCR validation of predicted helitrons}

The twelve representative maize inbred lines, including Mo17, Huangye4, W182bn, W153r, W117, W64a, Va102, Va35, N192, B73, B37 and B68, were chosen to validate the helitrons. Genomic DNA samples from each line were extracted from young seedling, according to the CTAB procedure [33]. Specific primers were designed in flanking upstream and downstream sequence of known elements. PCR reactions were performed using $1 \mathrm{ul}$ of the obtained DNA, 2 ul 10× PCR buffer, $0.75 \mathrm{ul} \mathrm{dNTPs}$ mixture $(2.5 \mathrm{mM}$ each), $1 \mathrm{ul}$ of primer mixture ( $5 \mathrm{uM}$ each), $0.25 \mathrm{ul}$ Taq polymerase, and distilled $\mathrm{H}_{2} \mathrm{O}$ was added to make up the final volume of $20 \mathrm{ul}$. The PCR conditions were $1 \mathrm{~min}$ at $95 \rrbracket$, then 35 cycles $95 \rrbracket$ for $45 \mathrm{~s}, \mathrm{x} \rrbracket(57 \rrbracket-62 \rrbracket)$ for $45 \mathrm{~s}$

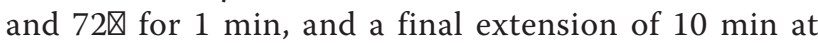
72区.

\section{Additional material}

Additional file 1: Figure S1. Verification of candidate helitrions. A Example of helitron inserted in repetitive sequences. B. Helitrons with multiple copies of high similarity can be verified each other by aligning their sequences together to determine their exact $5^{\prime}$ and $3^{\prime}$ boundaries.

Additional file 2: Figure S2. Verification of helitrons by PCR using 12 diversed inbred lines. Primers were designed in flanking inserted upstream and downstream sequences of putative helitrons. Vacant sites and occupied sites were displayed by different band sizes of PCR products. The names of the 12 inbred lines were from 1 to 12: Mo17, Huangye4, W182bn, W153r, W117, W64a, Va102, Va35, N192, B73, B37 and B68.

Additional file 3: Table S1. The location of the 1515 helAs in the maize genome

Additional file 4: Table S2. The location of the 134 helBs in the maize genome

Additional file 5: Figure S3. The sequence characteristics of 5'termini and $3^{\prime}$ - termini of helAs and helBs. A. 30 bp of 5'- termini of helAs; B. 40 bp of 3'-termini of helAs; C. 30 bp of 5'-termini of helBs; D. $40 \mathrm{bp}$ of $3^{\prime}$-termini of helBs.

Additional file 6: Table S3. The putative autonomous helitrons. The location of the putative autonomous helitrons in the maize genome.

Additional file 7: Table S4. The location of helitron_mc1 in the maize genome

Additional file 8: Table S5. The location of helitron_mc2 in the maize genome.
Additional file 9: Table S6. Gene fragments carried by helAs. Annotated protein of gene fragments carried by helAs.

Additional file 10: Table S7. Gene fragments carried by helBs Annotated protein of gene fragments carried by helBs.

Additional file 11: Table S8. Cross-referencing of helitrons between our result and Yang et al.'s result.

Additional file 12: Table S9. Cross-referencing of helitrons between our result and Du et al.'s result.

Additional file 13: Table S10. The characteristic of autonomous

helitrons in eukaryotes. "—_" indicated RPA, following the successive Rep motif and DNA helicase domain in two different ORF respectively. "-" indicated Rep motif, DNA helicase domain and RPA-DBD-A/-B/-C in their appropriate order in the same ORF.

\section{Acknowledgements}

This work is supported by the 973 project (2009CB118400) from the Ministry of Science and Technology.

\section{Authors' contributions}

J.L. designed the research. Y.D., X.L.,W.S. and L.S. did the data analysis. L.S., M. Z., H.Z. and Y.J wrote the PERL scripts. J.L. and Y.D. wrote the paper. All the authors have read and approved the final manuscript.

Received: 15 August 2011 Accepted: 17 December 2011

Published: 17 December 2011

\section{References}

1. Bennetzen JL, Ma J, Devos KM: Mechanisms of recent genome size variation in flowering plants. Ann Bot 2005, 95(1):127-132.

2. Kidwell MG, Lisch DR: Perspective: transposable elements, parasitic DNA and genome evolution. Evolution 2001, 55(1):1-24

3. Kapitonov W, Jurka J: Rolling-circle transposons in eukaryotes. Proc Natl Acad Sci USA 2001, 98(15):8714-8719.

4. Kapitonov W, Jurka J: Helitrons on a roll: eukaryotic rolling-circle transposons. Trends Genet 2007, 23(10):521-529.

5. Choi JD, Hoshino A, Park KI, Park IS, lida S: Spontaneous mutations caused by a Helitron transposon, Hel-It1, in morning glory, Ipomoea tricolor. Plant J 2007, 49(5):924-934

6. Kapitonov W, Jurka J: Molecular paleontology of transposable elements in the Drosophila melanogaster genome. Proc Natl Acad Sci USA 2003, 100(11):6569-6574.

7. Lal SK, Giroux MJ, Brendel V, Vallejos CE, Hannah LC: The maize genome contains a helitron insertion. Plant Cell 2003, 15(2):381-391.

8. Langdon T, Thomas A, Huang L, Farrar K, King J, Armstead I: Fragments of the key flowering gene GIGANTEA are associated with helitron-type sequences in the Pooideae grass Lolium perenne. BMC Plant Biol 2009, 9:70.

9. Poulter RT, Goodwin TJ, Butler MI: Vertebrate helentrons and other novel Helitrons. Gene 2003, 313:201-212.

10. Pritham EJ, Feschotte C: Massive amplification of rolling-circle transposons in the lineage of the bat Myotis lucifugus. Proc Natl Acad SCi USA 2007, 104(6):1895-1900.

11. Yang L, Bennetzen JL: Structure-based discovery and description of plant and animal Helitrons. Proc Natl Acad Sci USA 2009, 106(31):12832-12837.

12. Zhou Q, Froschauer A, Schultheis C, Schmidt C, Bienert GP, Wenning M, Dettai A, Volff JN: Helitron Transposons on the Sex Chromosomes of the Platyfish Xiphophorus maculatus and Their Evolution in Animal Genomes. Zebrafish 2006, 3(1):39-52.

13. Du C, Fefelova N, Caronna J, He L, Dooner HK: The polychromatic Helitron landscape of the maize genome. Proc Natl Acad Sci USA 2009, 106(47):19916-19921

14. Yang L, Bennetzen JL: Distribution, diversity, evolution, and survival of Helitrons in the maize genome. Proc Natl Acad Sci USA 2009, 106(47):19922-19927.

15. Morgante M, Brunner S, Pea G, Fengler K, Zuccolo A, Rafalski A: Gene duplication and exon shuffling by helitron-like transposons generate intraspecies diversity in maize. Nat Genet 2005, 37(9):997-1002. 
16. He L, Dooner HK: Haplotype structure strongly affects recombination in a maize genetic interval polymorphic for Helitron and retrotransposon insertions. Proc Natl Acad Sci USA 2009, 106(21):8410-8416.

17. Lai J, Li Y, Messing J, Dooner HK: Gene movement by Helitron transposons contributes to the haplotype variability of maize. Proc Natl Acad Sci USA 2005, 102(25):9068-9073.

18. Hollister JD, Gaut BS: Population and evolutionary dynamics of Helitron transposable elements in Arabidopsis thaliana. Mol Biol Evol 2007, 24(11):2515-2524.

19. Sweredoski M, DeRose-Wilson L, Gaut BS: A comparative computational analysis of nonautonomous helitron elements between maize and rice. BMC Genomics 2008, 9:467.

20. Jameson N, Georgelis N, Fouladbash E, Martens S, Hannah LC, Lal S: Helitron mediated amplification of cytochrome P450 monooxygenase gene in maize. Plant Mol Biol 2008, 67(3):295-304.

21. $\mathrm{Xu} \mathrm{JH}$, Messing J: Maize haplotype with a helitron-amplified cytidine deaminase gene copy. BMC Genet 2006, 7:52.

22. Ilyina TV, Koonin EV: Conserved sequence motifs in the initiator proteins for rolling circle DNA replication encoded by diverse replicons from eubacteria, eucaryotes and archaebacteria. Nucleic Acids Res 1992, 20(13):3279-3285.

23. Schnable PS, Ware D, Fulton RS, Stein JC, Wei F, Pasternak S, Liang C, Zhang J, Fulton L, Graves TA, et al: The B73 maize genome: complexity, diversity, and dynamics. Science 2009, 326(5956):1112-1115.

24. Du C, Caronna J, He L, Dooner HK: Computational prediction and molecular confirmation of Helitron transposons in the maize genome. BMC Genomics 2008, 9:51.

25. Brunner S, Pea G, Rafalski A: Origins, genetic organization and transcription of a family of non-autonomous helitron elements in maize. Plant J 2005, 43(6):799-810

26. Gupta S, Gallavotti A, Stryker GA, Schmidt RJ, Lal SK: A novel class of Helitron-related transposable elements in maize contain portions of multiple pseudogenes. Plant Mol Biol 2005, 57(1):115-127.

27. Wang $\mathrm{Q}$, Dooner HK: Remarkable variation in maize genome structure inferred from haplotype diversity at the bz locus. Proc Natl Acad Sci USA 2006, 103(47):17644-17649.

28. Feschotte C, Pritham EJ: A cornucopia of Helitrons shapes the maize genome. Proc Natl Acad Sci USA 2009, 106(47):19747-19748.

29. Tempel S, Nicolas J, El AA, Couee I: Model-based identification of Helitrons results in a new classification of their families in Arabidopsis thaliana. Gene 2007, 403(1-2):18-28.

30. Li Y, Dooner HK: Excision of Helitron transposons in maize. Genetics 2009, 182(1):399-402.

31. Baucom RS, Estill JC, Chaparro C, Upshaw N, Jogi A, Deragon JM, Westerman RP, Sanmiguel PJ, Bennetzen JL: Exceptional diversity, nonrandom distribution, and rapid evolution of retroelements in the $B 73$ maize genome. PLoS Genet 2009, 5(11):e1000732..

32. Tamura K, Dudley J, Nei M, Kumar S: MEGA4: Molecular Evolutionary Genetics Analysis (MEGA) software version 4.0. Mol Biol Evol 2007, 24(8):1596-1599.

33. Saghai-Maroof MA, Soliman KM, Jorgensen RA, Allard RW: Ribosomal DNA spacer-length polymorphisms in barley: mendelian inheritance, chromosomal location, and population dynamics. Proc Natl Acad Sci USA 1984, 81(24):8014-8018.

doi:10.1186/1471-2164-12-609

Cite this article as: Dong et al:: Structural characterization of helitrons and their stepwise capturing of gene fragments in the maize genome. BMC Genomics 2011 12:609.

\section{Submit your next manuscript to BioMed Central and take full advantage of:}

- Convenient online submission

- Thorough peer review

- No space constraints or color figure charges

- Immediate publication on acceptance

- Inclusion in PubMed, CAS, Scopus and Google Scholar

- Research which is freely available for redistribution

Submit your manuscript at www.biomedcentral.com/submit
Biomed Central 